This item was submitted to Loughborough's Research Repository by the author.

Items in Figshare are protected by copyright, with all rights reserved, unless otherwise indicated.

\title{
Validation of the GENEA accelerometer
}

PLEASE CITE THE PUBLISHED VERSION

http://dx.doi.org/10.1249/MSS.0b013e31820513be

\section{PUBLISHER}

Lippincott, Williams \& Wilkins (@ The American College of Sports Medicine)

\section{VERSION}

AM (Accepted Manuscript)

\section{LICENCE}

CC BY-NC-ND 4.0

\section{REPOSITORY RECORD}

Esliger, Dale W., Ann V. Rowlands, Tina L. Hurst, Michael Catt, Peter Murray, and Roger G. Eston. 2019. "Validation of the GENEA Accelerometer". figshare. https://hdl.handle.net/2134/15394. 
This item was submitted to Loughborough's Institutional Repository (https://dspace.lboro.ac.uk/) by the author and is made available under the following Creative Commons Licence conditions.

\section{creative
commons}

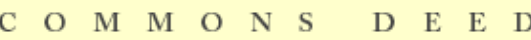

Attribution-NonCommercial-NoDerivs 2.5

You are free:

- to copy, distribute, display, and perform the work

Under the following conditions:

Attribution. You must attribute the work in the manner specified b the author or licensor.

Noncommercial. You may not use this work for commercial purposes.

No Derivative Works. You may not alter, transform, or build upon this work.

- For any reuse or distribution, you must make clear to others the license terms of this work.

- Any of these conditions can be waived if you get permission from the copyright holder.

Your fair use and other rights are in no way affected by the above.

This is a human-readable summary of the Leqal Code (the full license).

\section{Disclaimer 만}

For the full text of this licence, please go to: http://creativecommons.org/licenses/by-nc-nd/2.5/ 


\section{Validation of the GENEA accelerometer}

Running title: GENEA accelerometer

Dale W. Esliger ${ }^{1,4}$, Ann V. Rowlands ${ }^{1}$, Tina L. Hurst ${ }^{2}$, Michael Catt ${ }^{3,2}$, Peter Murray ${ }^{2}$, Roger G. Eston $^{1}$

${ }^{1}$ School of Sport and Health Sciences, St Luke's Campus, University of Exeter, Exeter, United

Kingdom

${ }^{2}$ Unilever Discover, Colworth, United Kingdom

${ }^{3}$ Institute for Ageing and Health, Newcastle University, Newcastle Upon Tyne, United Kingdom

${ }^{4}$ College of Kinesiology, University of Saskatchewan, Saskatoon, Saskatchewan, Canada

Funding: This research was supported by Unilever Discover

Address correspondence to:

Dale W. Esliger

College of Kinesiology

87 Campus Drive

Saskatoon, SK, Canada

S7N 5B2

dale.esliger@usask.ca

306-966-1085

306-966-6464 


\begin{abstract}
Purpose: The study aims were: 1) to assess the technical reliability and validity of the GENEA using a mechanical shaker, 2) to perform a GENEA value calibration to develop thresholds for sedentary, light, moderate, and vigorous intensity physical activity, and 3) to compare the intensity classification of the GENEA with two widely used accelerometers. Methods: 47 GENEAs were attached to a shaker and vertically accelerated generating 15 conditions of varying acceleration and/or frequency. Reliability was calculated using standard deviation and intra- and inter-instrument coefficient of variation, while validity was assessed via Pearson correlation with the shaker acceleration as the criterion. Next, 60 adults wore a GENEA on each wrist and on the waist (alongside an Actigraph and RT3 accelerometer) while completing 10-12 activity tasks. A portable metabolic gas analyzer provided the criterion measure of physical activity. Analyses involved the use of Pearson correlations to establish criterion and concurrent validity and ROC curves to establish intensity cut points. Results: The GENEA demonstrated excellent technical reliability $\left(\mathrm{CV}_{\text {intra }}=1.4 \% \mathrm{CV}_{\text {inter }}=2.1 \%\right)$ and validity $(\mathrm{r}=0.98 ; \mathrm{p}<0.001)$ using the mechanical shaker. The GENEA demonstrated excellent criterion validity using $\mathrm{VO}_{2}$ as the criterion (left wrist $\mathrm{r}=0.86$; right wrist $\mathrm{r}=0.83$; waist $\mathrm{r}=0.87$ ), on par with the waist-worn Actigraph and RT3. The GENEA demonstrated excellent concurrent validity compared to the Actigraph ( $\mathrm{r}=0.92)$ and the RT3 $(\mathrm{r}=0.97)$. The waist-worn GENEA had the greatest classification accuracy (Area Under the ROC curve; AUC=0.95), followed by the left $(0.93)$ then right wrist (0.90). The accuracy of the waist-worn GENEA was virtually identical to the Actigraph (AUC=0.94) and RT3 (0.95). Conclusions: The GENEA is a reliable and valid measurement tool capable of classifying the intensity of physical activity in adults.
\end{abstract}

Key Words: ACTIVITY MONITOR, MEASUREMENT, ACCELERATION, FREQUENCY, GRAVITY, RELIABILITY 


\section{INTRODUCTION}

Paragraph Number 1 The unequivocal link between physical activity and health has prompted exercise science and public health researchers, their learned societies, and their funders to search for better and more logistically feasible and objective tools to measure physical activity.

This focus has facilitated the development of many objective measurement technologies, such as accelerometers (described in detail elsewhere $(17,29)$ ), which provide robust and detailed physical activity information (4).

Paragraph Number 2 Although much progress has been made in the assessment of physical activity with accelerometers, there are a number of limitations that still need to be addressed. An important first step would be to convince manufacturers to abolish the practice of disguising raw acceleration outcomes via proprietary 'count' units as it hinders between model comparisons. Second, increased battery life and memory storage would benefit users that require higher resolution signals. This is especially important for multi-axis accelerometers and seismicbased technologies (26). However, a balance must be struck to ensure that the quest for richer data does not interfere with other more practical issues related to accelerometer size, weight, and functionality. For example, the IDEEA monitor (32) utilizes an array of wired accelerometers and as such is somewhat obtrusive. That said, there are some accelerometers such as the Sensor Wear Armband (7), the ActivPal (9), and the DynaPort (26), that seem to have balanced the desire for advanced measurement capabilities, such as activity classification, with feasibility issues (e.g., the desire for high resolution acceleration data and the need to remain unobtrusive). More recently, another accelerometer, the GENEA, has been developed.

Paragraph Number 3 This manuscript functions to introduce the GENEA, a novel acceleration sensor developed by Unilever Discover (Colworth, United Kingdom) and 
manufactured and distributed by Oralinsights Limited (Kimbolton, Cambridgeshire, United Kingdom). Therefore, the purpose of this study was threefold: 1) to perform a preliminary technical reliability and validity assessment of the GENEA using a mechanical shaker table, 2) to perform a value calibration of the GENEA to develop thresholds for sedentary, light, moderate, and vigorous intensity physical activity in adults, and 3) to compare the intensity classification of the GENEA with two widely used accelerometers.

\section{METHODS}

\section{Technical Reliability and Validity}

Paragraph Number 4 Accelerometers. The GENEA is a triaxial, $\pm 6 \mathrm{~g}$ seismic acceleration sensor (LIS3LV02DL; STMicroelectronics, Geneva, Switzerland). The small (36x30x12 LxWxH mm) and lightweight (16 grams), splashproof design of the GENEA allow it to be easily worn at multiple locations on the body (e.g., wrist, waist, ankle). The GENEA has 500 megabytes of memory to assist with the storage of the raw $80 \mathrm{~Hz}$ sampling frequency and can store $\sim 8$ days of data in raw mode with 12 bit resolution. Users have the ability to select userdefined sample frequencies ranging from 10-80 Hz. Using the GENEA software (version 1.487 update 531), via USB to PC connection, 47 GENEA accelerometers, were initialized to collect unfiltered, triaxial acceleration data at a sample rate of $80 \mathrm{~Hz}$.

Paragraph Number 5 Multi-axis shaking table. All technical reliability and validity testing was completed using a Multi-Axis Shaking Table (MAST) manufactured by Instron Structural Testing Systems (Buckinghamshire, United Kingdom) with an industrial Labtronic 8800 Digital Controller (Darmstadt, Germany). The MAST has been described in detail by Gizatullin and Edge (8). The MAST is designed to recreate spatial motion of the platform in three dimensions; however, only vertical motion was used in this preliminary technical reliability and validity 
study. Using a combination of command file and individually entered position command signals, the MAST was programmed to accurately and reliably oscillate the platform at the various testing conditions using a sinusoidal oscillation procedure. The testing conditions were restricted by the MAST stroke limits of approximately $\pm 75 \mathrm{~mm}$. The range of possible conditions of acceleration and frequency of oscillation are described by the equation: acceleration $\left(\mathrm{m} \cdot \mathrm{s}^{-2}\right)=\left(\operatorname{amplitude}(\mathrm{m}) \cdot\right.$ frequency $^{2}\left(\mathrm{rad} \cdot \mathrm{s}^{-1}\right)$. Fifteen different conditions were selected to produce a range of physiologically relevant accelerations from light to moderate to vigorous within the limitations of the MAST.

Paragraph Number 6 The GENEA accelerometers were secured/enclosed in a custom test jig (Figure 1, inset right) lined with high density foam to avoid potential vibration transference and bolted to the surface of the shaker table (Figure 1, inset left). Care was taken to ensure that the monitors were secured firmly so the vertical movement was along the y-axis of the GENEA. Next, the first test condition was programmed and the condition was executed accelerating all accelerometers simultaneously in the vertical plane for the 60 second test duration. All 15 conditions were completed in this fashion and when complete, the accelerometers were removed from the shaker plate and test jig.

Paragraph Number 7 Data Reduction. All 47 GENEA accelerometers were downloaded to the initialization PC resulting in the creation of $47 \mathrm{raw}, 80 \mathrm{~Hz}$ data files containing unfiltered, time and date stamped $\mathrm{x}, \mathrm{y}$, and $\mathrm{z}$ axis acceleration data (in gravitational units (g) complete with negative sign indicating directionality). These data were downloaded in comma separated values file format (.csv extension); however, the user may also (or instead) choose a binary format (.bin extension). Although both files contain the same data, the binary format is much more efficient at nearly 8 times smaller file size. Using the GENEA Post Processing software (version 1.2.1), 
the raw $80 \mathrm{~Hz}$ triaxial GENEA data were summarized into a signal magnitude vector (gravitysubtracted) $\left(\mathrm{SVM}_{\mathrm{gs}}\right)$ using 1 second epochs [see equation 1] (11). The resulting SI units for this outcome variable are $\mathrm{g} \cdot \mathrm{seconds}$.

$$
\mathrm{SVM}_{\mathrm{gs}}=\sum\left|\sqrt{x^{2}+y^{2}+z^{2}}-g\right|
$$

Equation 1.

where the correction for gravity was undertaken to focus the outcome variable on dynamic rather than static accelerations

Although not the focus of the present study, the post processing software does allow the user to summarize the raw triaxial signal using units of acceleration. This is achieved by using the mean signal magnitude vector over the user-defined epoch [see equation 2], rather than the integral measure of the SVM as outlined above (26).

$$
\operatorname{SVM}^{\bar{r}}=\sqrt{x^{2}+y^{2}+z^{2}}
$$

Equation 2.

as this is a mean rather than an cumulative sum, it retains its gravity-based acceleration units $(\mathrm{g})$; where $1 \mathrm{~g}=9.81 \mathrm{~m} \cdot \mathrm{s}^{-2}$

Next, data were imported into a customized spreadsheet application using the common epochby-epoch time stamp to align/synchronize the data across units and models (verified by crosscorrelation analyses). The recorded condition start and end times were identified and the middle 50 seconds of each condition were extracted for further analysis.

Paragraph Number 8 Statistical Analyses. For all test conditions the mean count output (50 replicate seconds) of each accelerometer for each condition was calculated. Intra- and interinstrument reliability was calculated using standard deviation and coefficient of variation $\left(\mathrm{CV}_{\text {intra }}\right.$ and $\mathrm{CV}_{\text {inter }}$ ). Pearson product-moment correlation was used to determine the criterion validity of 
the GENEA versus the MAST acceleration. All analyses were performed using SPSS version 15.0 for Windows (SPSS, Chicago, USA).

\section{Value Calibration}

Paragraph Number 9 Participants. Participant recruitment was initiated in February 2008 in an effort to obtain a convenience sample of 60 male and female volunteers aged 40-65 years. Two recruitment methods were used: an email to employees of the University of Exeter, Exeter, Devon, UK and an advertisement in a local newspaper. A health and fitness report and a $£ 20$ gift card were offered as incentive and honorarium respectively for participating in the study. Data collection was undertaken in March and April 2008 after which time 60 adults (62\% female) aged 40-63 years completed the study protocol. All participants were free from diagnosed disease and musculoskeletal injury and had no affirmative answers to the Physical Activity Readiness Questionnaire (PAR-Q). Written informed consent was obtained from each participant. The study was approved by the Ethics committee of the School of Sport and Health Sciences, University of Exeter.

Paragraph Number 10 Data Collection Procedures. Participants arrived at the laboratory having refrained from consuming nicotine, caffeine, or a large meal for at least two hours prior and exercise at least 6 hours prior to the appointment. As part of a larger battery of anthropometry and health-related fitness tests (peripheral to the present study), participants had their height (to the nearest $0.1 \mathrm{~cm}$ ) and body mass (to the nearest $0.1 \mathrm{~kg}$ ) measured using a Holtain stadiometer (Holtain, Crymych, Dyfedd, UK) and a Tanita, TBF-305 scale (Tanita UK Ltd., Middlesex, UK) respectively. Whole body bioelectrical impedance analysis (BodyStat 1500, BodyStat, Onchan, Isle of Man, United Kingdom) was also performed to determine percent body fat. Next, each participant was asked to complete an ordered series of 10-12 semi- 
structured activities in the laboratory and free-living environment. The lying activity was performed for 10 minutes while all other activities were performed for 4.5 minutes. In between each activity the participants were given at least 2 minutes rest to allow them to prepare for the next activity and for their metabolic rate (oxygen uptake) to recover to pre-activity levels. Throughout testing $\mathrm{VO}_{2}, \mathrm{VCO}_{2}$, and heart rate were measured by the Cosmed $\mathrm{K} 4 \mathrm{~b} 2$ (Rome, Italy) portable metabolic gas analysis system with heart rate receiver. Prior to each testing session the K4b2 was calibrated with gases of known concentration and the flow sensor calibration and environmental conditions updated. Lastly, the K4b2 and the computer running the software were time-synchronized with the Greenwich Mean Time server. The K4b2 has been shown to provide valid measurements of oxygen uptake across a range of exercise intensities (13). After each testing session the relative $\mathrm{VO}_{2}$ data were downloaded and stored on a PC for further analysis.

Paragraph Number 11 Accelerometer. Throughout testing, three GENEA accelerometers were worn, one on each of the left and right wrists (using simple watch straps, accelerometers positioned over the dorsal aspect of the wrists midway between the radial and ulnar styloid processes) and one on the waist (using a elasticized belt, accelerometer positioned over the right hip, mid-clavicular line landmarked by the supraspinale). The same three GENEAs were used by all 60 participants and were always positioned at the same sites for each. On the same belt, adjacent to the waist-worn GENEA, participants wore two peer accelerometers, a uniaxial Actigraph GT1M accelerometer with firmware version 3.0.0 (Actigraph, Pensacola, Florida, USA) and a triaxial RT3 accelerometer (Stayhealthy.com, Monrovia, California, USA). At the start of each testing session the accelerometers were time synchronized with the GMT time server and initialized to record acceleration data. The epoch length was set at $80 \mathrm{~Hz}, 1$ second, 
and 1 minute 3-axis for the GENEAs, Actigraph, and RT3's respectively; however, only the vector magnitude data from the RT3 were analyzed. After each testing session the accelerometer data were downloaded and stored on a PC for further analysis.

Paragraph Number 12 Data Analyses. Using the K4b2 software, the breath-by-breath $\mathrm{VO}_{2}$ data were filtered using 1 minute averaging. Using the GENEA Post Processing software (version 1.2.1), the raw $80 \mathrm{~Hz}$ triaxial GENEA data were summarized into $\mathrm{SVM}_{\mathrm{gs}}$ using 1 minute epoch intervals. The Actigraph data were also integrated to 1-minute epochs for further analysis. Custom spreadsheet applications were developed to temporally synchronize the minute-byminute data from the $\mathrm{K} 4 \mathrm{~b} 2$ with the data from the three accelerometer models. The combined data set was then plotted to facilitate the selection of the minute of accelerometer data that coincided with steady-state $\dot{\mathrm{V}} \mathrm{O}_{2}$ for each of the activities performed. Ideally, and in most cases, this was the $4^{\text {th }}$ minute of each activity; however, in a minority of cases the $3^{\text {rd }}$ minute was selected as it better represented steady-state due to occasional time synchronization issues (the $10^{\text {th }}$ or $9^{\text {th }}$ minute for the lying activity). The relative $\dot{\mathrm{V}} \mathrm{O}_{2}$ and accelerometer data corresponding to the selected minute were exported to SPSS version 15.0 for Windows (SPSS, Chicago, USA) for further analysis. Pearson correlations were calculated between each accelerometer output and $\dot{\mathrm{V}} \mathrm{O}_{2}$.

Paragraph Number 13 The $\dot{\mathrm{VO}}_{2}$ data were converted to METS using the standard conversion of $1 \mathrm{MET}=3.5 \mathrm{ml} \cdot \mathrm{kg}^{-1} \cdot \mathrm{min}^{-1}$ and then coded into one of four absolute intensity categories: sedentary (<1.5 METS), light (1.5-3.99 METS), moderate (4.00-6.99 METS), or vigorous (7+ METS) activity. The impetus for using 4 and 7, rather than 3 and 6 MET thresholds to mark moderate and vigorous intensity physical activity follows the rationale described in a review by Shephard (23). Citing work by Porcari et al. (19), Shephard noted that 
those deriving health benefits from lifestyle activities tended to be sedentary, obese, and elderly people, those for whom a given absolute intensity of effort such as brisk walking develops a substantial relative intensity of effort. Simply put, because the present sample were sedentary/unfit their physical activity energy expenditure for a given absolute intensity effort such as a brisk walk is likely to be higher than for fitter individuals. Therefore, developing cut points on an unfit sample using 3 and 6 MET thresholds would result in an increased chance of erroneously categorizing fitter individuals as active rather than inactive. As this was the case in the present study, the higher absolute intensity cut points of 4 and 7 METS were chosen.

Paragraph Number 14 Next, the accelerometer data were recoded to create binary indicator variables ( 0 or 1$)$ to facilitate the Receiver Operator Characteristic (ROC) curve analyses. For sedentary, this corresponded to sedentary activities versus more than sedentary activities. For moderate intensity, this corresponded to less than moderate activities versus moderate to vigorous activities. For vigorous intensity, this corresponded to vigorous activities versus less than vigorous activities. Next, the binary coded accelerometer data were exported to GraphPad Prism 4.00 for Windows, (GraphPad Software, San Diego, USA) to undergo the ROC curve analyses.

Paragraph Number 15 A ROC curve is a graphical technique for describing and comparing the accuracy of diagnostic tests. In the present application, ROC analysis is used to examine the potential of using thresholds within the GENEA, Actigraph, and RT3 data to discriminate between four activity intensity categories. As Jago et al. (10) described, ROC analysis is a means to evaluate and visualize the sensitivity [true positives/(true positives + false negatives)] and specificity [true negatives/(true negatives + false positives)] of tests. The ROC curve is simply a plot of the sensitivity of a test on the y-axis versus its 1-specificity (i.e. false 
positive fraction on the $\mathrm{x}$-axis). Each possible threshold value corresponds to a point on the ROC curve. The upper-left corner [the point $(0,1)]$ represents perfect classification, and the diagonal line represents the strategy of randomly guessing. Sensitivity is maximized by correctly identifying at or above the threshold for intensity, whereas specificity is maximized by correctly excluding activities below the threshold for intensity. Similar to the methods of Evenson et al. (6), the cut points at which sensitivity and specificity were both maximized were identified.

\section{RESULTS}

\section{Technical Reliability and Validity}

Paragraph Number 16 All 47 GENEA accelerometers successfully initialized, collected, and downloaded data. The mean $\mathrm{SMV}_{\mathrm{gs}}(\mathrm{g} \cdot$ seconds) for the GENEA accelerometers displayed a linear trajectory across all 15 testing conditions when organized from low to high acceleration values (Figure 1, main). The average intra- and inter-instrument reliability of the 47 GENEA accelerometers across the 15 conditions was $\mathrm{CV}_{\text {intra }}=1.8 \%$ and $\mathrm{CV}_{\text {inter }}=2.4 \%$ respectively. The criterion validity for the GENEA versus the MAST acceleration for all 15 test conditions was excellent $(\mathrm{r}=0.97 ; \mathrm{p}<0.001)$.

\section{Value Calibration}

Paragraph Number 17 Descriptive characteristics of the participants are shown in Table 2. The BMI data are a testament to the fact that this is a convenience sample as the participants are leaner than the general population. In fact, the prevalence of obesity among the group was $4 \%$ for men and $5 \%$ for women, much lower than the $23 \%$ and $25 \%$ found in the general population (21). However, the number of left handed participants in this study (12\%) matched well the $11 \%$ prevalence of left handedness in the UK population (14). 
Paragraph Number 18 Table 3 compares the average METS and the position-specific GENEA results. As expected, the activities in the posture grouping were the lowest intensity while the optional running activities were the highest which was reflected in the $\dot{\mathrm{V}} \mathrm{O}_{2}$ and the accelerometer output. The fact that the slow treadmill walking, an activity intended to be light intensity, resulted in an average 3.88 METS response helps to justify the decision to utilize the higher absolute intensity cut points of 4 and 7 METS to distinguish moderate and vigorous intensity activity in this sample. Comparing the different wear positions of the GENEA showed that data for the left and right wrists were similar, while the mean GENEA data collected at the waist were lower as were the standard deviations. Comparative data from the waist-worn Actigraph and RT3 are also displayed in Table 3.

Paragraph Number 19 Using relative $\mathrm{VO}_{2}$ as the criterion, the GENEA demonstrated excellent criterion validity across all activities (left: $r=0.86$; right: $r=0.83$, waist: $r=0.87$ ), performing as well at the waist as the Actigraph GT1M $(r=0.86)$ and RT3 $(r=0.88)$. The GENEA also demonstrated excellent concurrent validity compared to the Actigraph GT1M ( $\mathrm{r}=$ 0.92) and the RT3 (0.97) accelerometers. Device-specific scatter plots in Figure 2 provide a graphical comparison of criterion validity (i.e., METS versus each accelerometer's output) across the varying activity conditions.

Paragraph Number 20 Table 4 outlines the results of the ROC curve analysis for the GENEA. Across all three wear positions, discrimination of sedentary behavior was almost perfect, with the area under the ROC curve ranging from 0.97-0.98. On account of reduced specificity, the discrimination of moderate activity was slightly less precise, ranging from 0.840.93. Meanwhile, it was the reduced sensitivity of vigorous intensity cut points that compromised the discrimination of vigorous activity which ranged from 0.89-0.92. Overall, the 
waist-worn GENEA had the greatest classification accuracy (0.95), followed by the left $(0.93)$ then right wrist (0.90). Interestingly, the GENEA on the right wrist was unable to discriminate between light and moderate intensity categories. The overall ability to discriminate between sedentary, light, moderate, and vigorous intensity physical activity of the waist-worn GENEA was virtually identical to that of the Actigraph GT1M (0.94) and the RT3 (0.95) (data not shown).

\section{DISCUSSION}

\section{Technical Reliability and Validity}

Paragraph Number 21 Researchers have used various mechanical apparatuses to oscillate accelerometers in various axes in an effort to assess reliability. These apparatuses allow the researcher to control the magnitude of the acceleration being imparted as well as the frequency of the oscillation, two key variables that contribute to the accelerometer's output. Mechanical setups, by virtue of the precise control of the experimental conditions, are able to determine the variability attributed solely to the accelerometer. Examples include turntables (15), rotating wheel setups $(2,16)$, vibration tables $(20)$, and various types of mechanical shakers $(5,12,22$, 24, 26). This type of testing is important because if the measurement error intrinsic to the accelerometer is found to be small, then focus can shift to other sources of variation (e.g., position worn on the body, variation over time (e.g., day-to-day, week-to-week, season-toseason) (15).

Paragraph Number 22 This is the first study to evaluate the technical reliability and validity of the newly introduced GENEA accelerometer. Utilizing 15 testing conditions across a range of accelerations and frequencies, the GENEA was found to be highly reliable with mean intra- and inter-instrument coefficients of variation of 1.8 and $2.4 \%$ respectively. Likewise, the 
GENEA was found to have excellent criterion validity when compared to the MAST acceleration $(\mathrm{r}=0.97)$. Two thirds of the above-mentioned technical reliability studies focus on Actigraph model accelerometers and as such, it is a logical comparator for the GENEA. Out of six studies, the current GENEA results demonstrate the highest technical reliability with the next best performer coming in a recent study on the Actigraph GT1M $\left(\mathrm{CV}_{\text {intra }}=2.9 \%\right.$ and $\left.\mathrm{CV}_{\text {inter }}=3.5 \%\right)$ (24). In terms of criterion validity with the MAST, the only relevant comparison comes from a study on the Actigraph 7164 (2) where the validity coefficient was identical to the present study.

\section{Value Calibration}

Paragraph Number 23 This is the first study to develop accelerometer cut points for both wrist and waist-worn GENEA accelerometers that reflect sedentary, light, moderate, and vigorous intensity physical activity in adults. The delineation of intensity of physical activity from accelerometer data aids in the understanding of the relationship between health-related physical activity and potential predictor variables such as adiposity, age, health status, and ethnicity (10). The process used to convert raw accelerometer data into more meaningful and interpretable units is generally referred to as "value calibration" (30). Although raw accelerometer data provides an indicator of overall movement, a fundamental research challenge has been to determine how to equate it to more meaningful indicators, such as energy expenditure or time spent at given activity intensity. Being able to identify the amount of time spent in a range of intensity categories is useful given the fact that numerous international physical activity guidelines recommend various amounts of time be spent in specific intensity categories (27).

Paragraph Number 24 In the present study the GENEA demonstrated high levels of criterion validity (with an average correlation with METS across wear positions of $\mathrm{r}=0.85$ ) across 10-12 static and dynamic physical activities. In fact, the criterion validity of the GENEA 
was virtually identical to that of the Actigraph and RT3. Similarly high levels of criterion validity were shown by Bouten et al. (1) during the development of their novel triaxial accelerometer. The GENEA also demonstrated high levels of concurrent validity compared to the Actigraph GT1M and the RT3, with the highest correlations between the triaxial devices. These data illustrate that the GENEA is comparable to peer accelerometers, a result that is confirmed in the graphical representation of the data in Figure 2. However, a closer look suggests that the GENEA data are less variable than the RT3 and Actigraph across the intensity spectrum. Although speculative, the tighter clustering of data, within activities may provide greater success for the GENEA when pursuing alternative analytical approaches. For example, the tighter clustering of data within activities in the GENEA should allow for more optimal activity classification (i.e., the determination of the mode of physical activity).

Paragraph Number 25 The GENEA $\mathrm{SVM}_{\mathrm{gs}}(\mathrm{g} \cdot$ minutes) cut points established in this study demonstrated excellent accuracy for classifying physical activity intensity across the intensity spectrum. Because this is the first article to report cut points for the GENEA, it is not possible to compare these values with other studies. Although the accuracy of the GENEA was greatest at the waist, it also performed well at the wrist, with the left wrist being more accurate than the right. The diminished accuracy experienced on the right wrist was likely due to differences in participant handedness; that is, extraneous movements recorded during the activity conditions were more likely to occur on the right wrist (e.g., scratching, adjusting clothing/glasses, hand gestures). However, given small number of left handed participants $(\mathrm{n}=5)$, testing of the hypothesis was untenable. However, it may be that activity signatures obtained from the right wrist (dominant for over $80 \%$ of the population) may prove more useful than the left (non-dominant) wrist. Future research should specifically address this issue so that users, 
especially left handed users, may know if wearing the GENEA on the right hand is possible. The small size of the GENEA and the fact that it can be positioned at the wrist, a location intuitively less obtrusive than the waist, may help rectify issues related to participant compliance in future studies and may allow for studies with extended physical activity monitoring periods (28).

Paragraph Number 26 Strengths and Limitations. Several strengths are noteworthy in the present study. The first strength was the use of a mechanical shaker to perform a preliminary technical reliability and validity assessment on the GENEA. The use of this well controlled experimental setup allowed for a robust assessment of this new technology. Next, the GENEA output was collected, analyzed, and presented in a non-proprietary SI unit and as such, can be compared with other devices that retain/employ classical physics based measurements. Next, ROC curve analysis was chosen to determine the physical activity intensity cut points which is known to be superior to previous accelerometer calibration methods that employed linear regression approaches (see Jago et al. (10) for a more thorough discussion on this topic). To date, only four studies have employed ROC curve analysis to generate cut points, and these were done on children $(3,6,10,31)$. A further strength of the ROC curve analyses was that the cut points were chosen to optimize the balance between sensitivity and specificity (i.e., point nearest 0,1 on the ROC curve) which in all analyses also coincided with the Youden index, ensuring the optimality of the cut points. The intuitive interpretation of the Youden index is that it is the point on the curve farthest from chance (18). Finally, the comparison to two widely used peer technologies also strengthens the study as does the fact that energy expenditure $\left(\mathrm{ml} \cdot \mathrm{kg}^{-1} \cdot \mathrm{min}^{-1}\right)$, rather than direct observation was used to provide a continuous, objective, and physiologically meaningful dependent variable with which to compare the GENEA data (25). 
Paragraph Number 27 Several limitations of this study should be acknowledged. First, the preliminary technical reliability and validity assessment was only performed on one axis, the vertical, leaving the performance of the other two axes uncharacterized. Next, the comparison of the GENEA would have been more appropriate with the triaxial Actigraph GT3X rather than the GT1M accelerometer; however, at the time of this study the newer Actigraphs were not part of our accelerometer inventory. Next, although our sample includes men and women of differing ages and body size, our population may not be representative. Larger and more variable samples (e.g., more left handed, more overweight and obese participants) are needed to determine if these factors might modify our findings. In addition, the activity conditions selected in this study may not represent the full complement of activities undertaken by a population; therefore, caution must be used when generalizing these results to other groups. Finally, cross-validation of the GENEA physical activity intensity cut points by other research groups in different adult populations is warranted.

\section{CONCLUSION}

Paragraph Number 28 The GENEA demonstrated excellent technical reliability and validity and excellent criterion validity compared to $\mathrm{VO}_{2}$. The GENEA cut points established in this study can be used to estimate the time spent in sedentary, light, moderate, and vigorous intensity physical activity in adults. As a result, the GENEA has established itself as an objective and feasible measurement tool, comparable to other peer accelerometers, such as the Actigraph GT1M and RT3 with the potential of offering advanced measurement features in the future. Additional work is warranted to further refine the adult cut points and to develop cut points for other age groups in an effort to calibrate the GENEA across the lifespan. Given that the high resolution sampling of the GENEA allows for advanced data mining (e.g., extraction of 
frequency domain features), future research should strive to classify/recognize activities of daily living.

\section{Acknowledgments}

This research was supported by a grant from Unilever Discover, Colworth, Bedford, UK. The authors would like to thank the participants, the graduate research assistants, and the administrative staff of the School of Sport and Health Sciences for their dedication to this research project.

\section{Conflict of Interest}

The results of the present study do not constitute endorsement by the authors or ACSM of the products described in this paper. This research was funded by an unrestricted research grant awarded by Unilever Discover to the School of Sport and Health Sciences, University of Exeter. The grant funded the salary of DE and small portions of the salaries of AR and RE. At the time of this research $\mathrm{TH}$ and MC were both employed by Unilever Discover. However, at the time of manuscript submission, $\mathrm{MC}$ and $\mathrm{DE}$ have no further relationship with Unilever Discover or any of its operating companies. None of the authors have a conflict of interest with Oralinsights, the manufacturer of the technology on which this manuscript is based. 


\section{References}

1. Bouten CV, Koekkoek KT, Verduin M, Kodde R, Janssen JD. A triaxial accelerometer and portable data processing unit for the assessment of daily physical activity. IEEE Trans Biomed Eng. 1997;44(3):136-47.

2. Brage S, Brage N, Wedderkopp N, Froberg K. Reliability and validity of the Computer Science and Applications accelerometer in a mechanical setting. Measurement in Physical Education and Exercise Science. 2003;7(2):101-19.

3. Chu EY, McManus AM, Yu CC. Calibration of the RT3 accelerometer for ambulation and nonambulation in children. Med Sci Sports Exerc. 2007;39(11):2085-91.

4. Esliger DW and Tremblay MS. Physical activity and inactivity profiling: the next generation. Can J Public Health. 2007;98 Suppl 2:S195-S207.

5. Esliger DW and Tremblay MS. Technical reliability assessment of three accelerometer models in a mechanical setup. Med Sci Sports Exerc. 2006;38(12):2173-81.

6. Evenson KR, Catellier DJ, Gill K, Ondrak KS, McMurray RG. Calibration of two objective measures of physical activity for children. J Sports Sci. 2008;1-9.

7. Fruin ML and Rankin JW. Validity of a multi-sensor armband in estimating rest and exercise energy expenditure. Med Sci Sports Exerc. 2004;36(6):1063-9.

8. Gizatullin AO and Edge KA. Adaptive control for a multi-axis hydraulic test rig. Proceedings of the Institution of Mechanical Engineers, Part I: Journal of Systems and Control Engineering. 2007;221(2):183-98.

9. Grant PM, Ryan CG, Tigbe WW, Granat MH. The validation of a novel activity monitor in the measurement of posture and motion during everyday activities. Br J Sports Med. 2006;40(12):992-7.

10. Jago R, Zakeri I, Baranowski T, Watson K. Decision boundaries and receiver operating characteristic curves: new methods for determining accelerometer cutpoints. J Sports Sci. 2007;25(8):937-44. 
11. Karantonis DM, Narayanan MR, Mathie M, Lovell NH, Celler BG. Implementation of a real-time human movement classifier using a triaxial accelerometer for ambulatory monitoring. IEEE Trans Inf Technol Biomed. 2006;10(1):156-67.

12. Krasnoff JB, Kohn MA, Choy FK, Doyle J, Johansen K, Painter PL. Interunit and intraunit reliability of the RT3 triaxial accelerometer. J Phys Act Health. 2008;5(4):527-38.

13. McLaughlin JE, King GA, Howley ET, Bassett DR, Jr., Ainsworth BE. Validation of the COSMED K4 b2 portable metabolic system. Int J Sports Med. 2001;22(4):280-4.

14. McManus IC and Hartigan A. Declining left-handedness in Victorian England seen in the films of Mitchell and Kenyon. Curr Biol. 2007;17(18):R793-R794.

15. Metcalf BS, Curnow JS, Evans C, Voss LD, Wilkin TJ. Technical reliability of the CSA activity monitor: The EarlyBird Study. Med Sci Sports Exerc. 2002;34(9):1533-7.

16. Moeller NC, Korsholm L, Kristensen PL, Andersen LB, Wedderkopp N, Froberg K. Unit-specific calibration of Actigraph accelerometers in a mechanical setup - is it worth the effort? The effect on random output variation caused by technical inter-instrument variability in the laboratory and in the field. BMC Med Res Methodol. 2008;8:19.

17. Montoye HJ, Kemper HCG, Saris WHM, Washburn RA. Measuring Physical Activity and Energy Expenditure. Champaign, IL: Human Kinetics; 1996.

18. Perkins NJ and Schisterman EF. The inconsistency of "optimal" cutpoints obtained using two criteria based on the receiver operating characteristic curve. Am J Epidemiol. 2006;163(7):670-5.

19. Porcari J, McCarron R, Kline G, Freedson PS, Ward A, Ross JA, Rippe JM. Is fast walking an adequate aerobic training stimulus for 30- to 69-year-old men and women? Physician Sportsmed. 1987;15(2):119-29.

20. Powell SM, Jones DI, Rowlands AV. Technical variability of the RT3 accelerometer. Med Sci Sports Exerc. 2003;35(10):1773-8.

21. Rennie KL and Jebb SA. Prevalence of obesity in Great Britain. Obes Rev. 2005;6(1):112. 
22. Rothney MP, Apker GA, Song Y, Chen KY. Comparing the performance of three generations of ActiGraph accelerometers. J Appl Physiol. 2008;105(4):1091-7.

23. Shephard RJ. Absolute versus relative intensity of physical activity in a dose-response context. Med Sci Sports Exerc. 2001;33(6 Suppl):S400-S418.

24. Silva P, Esliger DW, Welk GJ, Mota J. Technical reliability assessment of the Actigraph GT1M accelerometer. Measurement in Physical Education and Exercise Science. 2010;14:79-91.

25. Sirard JR, Trost SG, Pfeiffer KA, Dowda M, Pate RR. Calibration and evaluation of an objective measure of physical activity in preschool children. Journal of Physical Activity and Health. 2005;3:345-57.

26. Van Hees V, Slootmaker SM, De GG, Van MW, Van Lummel RC. Reproducibility of a triaxial seismic accelerometer (DynaPort). Med Sci Sports Exerc. 2009;41(4):810-7.

27. Warburton DE, Katzmarzyk PT, Rhodes RE, Shephard RJ. Evidence-informed physical activity guidelines for Canadian adults. Can J Public Health. 2007;98 Suppl 2:S16-S68.

28. Ware LJ, Hurling R, Bataveljic O, Fairley BW, Hurst TL, Murray P, Rennie KL, Tomkins CE, Finn A, Cobain MR, Pearson DA, Foreyt JP. Rates and determinants of uptake and use of an internet physical activity and weight management program in office and manufacturing work sites in England: cohort study. J Med Internet Res. 2008;10(4):e56.

29. Welk G. Physical Activity Assessments for Health-Related Research. Champaign, IL: Human Kinetics; 2002.

30. Welk GJ. Principles of design and analyses for the calibration of accelerometry-based activity monitors. Med Sci Sports Exerc. 2005;37(11 Suppl):S501-S511.

31. Welk GJ, Eisenmann JC, Schaben J, Trost SG, Dale D. Calibration of the biotrainer pro activity monitor in children. Pediatr Exerc Sci. 2007;19(2):145-58.

32. Zhang K, Werner P, Sun M, Pi-Sunyer FX, Boozer CN. Measurement of human daily physical activity. Obes Res. 2003;11(1):33-40. 


\section{Figure Captions}

Figure 1. Inset right: Overhead view of GENEA accelerometers positioned in the test jig; Inset left: Multi-Axis Shaker Table (MAST) complete with the test jig secured in place; Main Figure: Comparison of a typical output trace from one GENEA accelerometer across the 15 test conditions

Notes: i) the trace represents the middle 50 seconds of each of the 15 conditions

Figure 2. Relationship between physical activity intensity (METS) versus the outputs from the waist worn GENEA SVMgs (g · minutes), RT3 (counts/minute) and Actigraph (counts/min) accelerometers across the 16 activity conditions 
Table 1. Reliability results for the GENEA for all 15 test conditions

\begin{tabular}{|c|c|c|c|c|c|c|c|}
\hline \multicolumn{2}{|c|}{ Acceleration } & \multirow{2}{*}{$\begin{array}{c}\text { Frequency } \\
(\mathrm{Hz})\end{array}$} & \multirow{2}{*}{$\begin{array}{c}\text { Amplitude } \\
\text { (m) }\end{array}$} & \multicolumn{2}{|c|}{$\begin{array}{c}\text { GENEA SVMgs } \\
(\mathrm{g} \cdot \text { seconds })\end{array}$} & \multirow{2}{*}{$\begin{array}{c}\begin{array}{c}\text { CV intra } \\
\%\end{array} \\
\text { GENEA }\end{array}$} & \multirow{2}{*}{$\begin{array}{c}\begin{array}{c}\text { CV inte } \\
\%\end{array} \\
\text { GENEA }\end{array}$} \\
\hline$g$ & $\left(\mathrm{~m} \cdot \mathrm{s}^{-2}\right)$ & & & Mean & SD & & \\
\hline 0.05 & 0.49 & 1.0 & 0.0124 & 3 & 0.1 & 3.9 & 5.3 \\
\hline 0.22 & 2.16 & 1.0 & 0.0547 & 11 & 0.2 & 1.7 & 2.0 \\
\hline 0.46 & 4.51 & 3.0 & 0.0127 & 23 & 0.2 & 0.8 & 1.4 \\
\hline 0.46 & 4.51 & 2.5 & 0.0183 & 24 & 0.2 & 0.9 & 1.4 \\
\hline 0.48 & 4.71 & 2.0 & 0.0298 & 25 & 0.3 & 1.0 & 1.4 \\
\hline 0.49 & 4.81 & 1.5 & 0.0541 & 25 & 0.3 & 1.1 & 1.6 \\
\hline 0.79 & 7.75 & 5.0 & 0.0079 & 41 & 0.4 & 1.0 & 1.5 \\
\hline 0.91 & 8.93 & 3.0 & 0.0251 & 46 & 0.4 & 0.8 & 1.3 \\
\hline 1.25 & 12.26 & 4.5 & 0.0153 & 57 & 0.8 & 1.5 & 2.1 \\
\hline 1.29 & 12.65 & 4.0 & 0.0200 & 58 & 0.8 & 1.3 & 2.0 \\
\hline 1.31 & 12.85 & 3.5 & 0.0266 & 59 & 1.2 & 2.1 & 2.6 \\
\hline 1.38 & 13.54 & 3.0 & 0.0381 & 60 & 0.6 & 1.0 & 1.8 \\
\hline 1.68 & 16.48 & 4.5 & 0.0206 & 64 & 2.2 & 3.4 & 3.7 \\
\hline 1.84 & 18.05 & 3.0 & 0.0508 & 66 & 0.9 & 1.3 & 2.2 \\
\hline \multirow[t]{2}{*}{2.52} & 24.72 & 4.5 & 0.0309 & 84 & 4.3 & 5.1 & 5.3 \\
\hline & & \multicolumn{4}{|c|}{ Average variability across all 15 test conditions } & 1.8 & 2.4 \\
\hline
\end{tabular}


Table 2. Descriptive characteristics of the study participants

\begin{tabular}{lcccccccc}
\hline Gender & $\begin{array}{c}\text { Sample } \\
\text { Size* }\end{array}$ & Age & $\begin{array}{c}\text { Left } \\
\text { Handed }\end{array}$ & $\begin{array}{c}\text { Right } \\
\text { Handed }\end{array}$ & $\begin{array}{c}\text { Height } \\
(\mathbf{c m})\end{array}$ & $\begin{array}{c}\text { Weight } \\
(\mathbf{k g})\end{array}$ & $\begin{array}{c}\text { BMI } \\
\left(\mathbf{k g} \cdot \mathbf{m}^{-2}\right)\end{array}$ & $\begin{array}{c}\text { Body Fat } \\
(\boldsymbol{\%})\end{array}$ \\
\hline Male & 23 & $48.9(6.8)$ & 1 & 22 & $176.2(6.2)$ & $80.6(11.6)$ & $25.9(2.7)$ & $20.7(3.4)$ \\
Female & 37 & $49.6(6.4)$ & 4 & 33 & $162.8(5.4)$ & $62.9(8.4)$ & $23.8(3.5)$ & $32.8(6.6)$ \\
Overall & 60 & $49.4(6.5)$ & 5 & 55 & $167.9(8.7)$ & $69.7(13.0)$ & $24.6(3.4)$ & $28.2(8.1)$ \\
\hline
\end{tabular}

*for the measurement of body fat, there were 2 less participants ( 1 male and 1 female). 
Table 3. Average intensity (METS) and average left, right, and waist positioned GENEA output by activity

GENEA

Actigraph

RT3

(SVMgs $(\mathrm{g} \cdot$ minute $)$

\begin{tabular}{|c|c|c|c|c|c|c|c|c|c|c|c|c|c|c|}
\hline \multirow[b]{3}{*}{ Activity } & \multirow[b]{3}{*}{ Grouping } & \multirow{3}{*}{$\mathbf{N}^{*}$} & \multirow{2}{*}{\multicolumn{2}{|c|}{ METS }} & \multirow{2}{*}{\multicolumn{2}{|c|}{ Left Wrist }} & \multicolumn{4}{|c|}{$(\mathrm{SVMgs}(\mathrm{g} \cdot \mathrm{minutes})$} & \multicolumn{2}{|c|}{ (Counts/Minute) } & \multirow{2}{*}{\multicolumn{2}{|c|}{$\frac{(\text { VM Counts/Minute) }}{\text { Waist }}$}} \\
\hline & & & & & & & Right & Nrist & & & & & & \\
\hline & & & Mean & $\mathrm{SD}$ & Mean & $\mathrm{SD}$ & Mean & SD & Mean & $\mathrm{SD}$ & Mean & $\mathrm{SD}$ & Mean & SD \\
\hline Lateral Recumbent (lying on side) & \multirow{3}{*}{$\begin{array}{l}\text { Posture } \\
\text { (all) }\end{array}$} & 53 & 0.94 & 0.23 & 63 & 55 & 143 & 53 & 34 & 17 & 0 & 0 & 4 & 11 \\
\hline Seated Computer Work & & 55 & 1.22 & 0.29 & 185 & 86 & 242 & 67 & 48 & 33 & 1 & 3 & 9 & 21 \\
\hline Standing & & 55 & 1.13 & 0.25 & 100 & 66 & 103 & 177 & 48 & 15 & 0 & 0 & 3 & 9 \\
\hline Window Washing & \multirow{4}{*}{$\begin{array}{l}\text { Lifestyle } \\
(2 \text { of } 4)^{\dagger}\end{array}$} & 31 & 3.37 & 1.06 & 469 & 693 & 1808 & 708 & 185 & 61 & 138 & 177 & 463 & 344 \\
\hline Washing Dishes & & 24 & 2.35 & 0.45 & 634 & 177 & 768 & 239 & 132 & 164 & 51 & 80 & 160 & 74 \\
\hline Shelf Stacking & & 30 & 4.19 & 0.98 & 884 & 224 & 940 & 202 & 214 & 72 & 2620 & 1933 & 421 & 297 \\
\hline Sweeping & & 23 & 3.39 & 0.67 & 878 & 264 & 976 & 289 & 187 & 48 & 465 & 355 & 356 & 96 \\
\hline Slow Treadmill Walk $\left(4 \mathrm{~km} \cdot \mathrm{hr}^{-1}\right)$ & \multirow{3}{*}{$\begin{array}{l}\text { Ambulatory } \\
\text { (all) }\end{array}$} & 54 & 3.88 & 0.69 & 864 & 195 & 847 & 232 & 856 & 221 & 2323 & 643 & 1267 & 318 \\
\hline Medium Treadmill Walk $\left(5 \mathrm{~km} \cdot \mathrm{hr}^{-1}\right)$ & & 55 & 4.59 & 0.79 & 1177 & 299 & 1204 & 357 & 1264 & 214 & 3745 & 656 & 1659 & 331 \\
\hline Brisk Treadmill Walk $\left(6 \mathrm{~km} \cdot \mathrm{hr}^{-1}\right)$ & & 55 & 5.88 & 0.98 & 1506 & 400 & 1513 & 350 & 1815 & 272 & 4917 & 940 & 2228 & 450 \\
\hline Stair Ascent/Descent $\left(80\right.$ steps $\left.\cdot \mathrm{min}^{-1}\right)$ & Stairs (all) & 55 & 6.19 & 1.10 & 836 & 620 & 794 & 597 & 697 & 127 & 2427 & 780 & 811 & 208 \\
\hline Slow Treadmill Run $\left(8 \mathrm{~km} \cdot \mathrm{hr}^{-1}\right)$ & \multirow{3}{*}{$\begin{array}{l}\text { Ambulatory } \\
(1 \text { of } 3)^{\ddagger}\end{array}$} & 18 & 11.13 & 1.38 & 3858 & 588 & 4087 & 798 & 3553 & 593 & 8936 & 1794 & 4578 & 799 \\
\hline Medium Treadmill Run $\left(10 \mathrm{~km} \cdot \mathrm{hr}^{-1}\right)$ & & 14 & 12.00 & 1.24 & 5345 & 1073 & 4915 & 787 & 4043 & 325 & 8904 & 1826 & 5419 & 423 \\
\hline Fast Treadmill Run $\left(12 \mathrm{~km} \cdot \mathrm{hr}^{-1}\right)$ & & 5 & 13.61 & 0.60 & 5096 & 2339 & 5358 & 2460 & 4066 & 299 & 6982 & 3233 & 6093 & 674 \\
\hline Brisk Free-living Walk (6 km·hr-1) & $\begin{array}{c}\text { Ambulatory } \\
\text { (all) }\end{array}$ & 40 & 5.76 & 0.94 & 1433 & 171 & 1474 & 216 & 1769 & 227 & 4912 & 748 & 2434 & 559 \\
\hline Medium Free-living Run $\left(10 \mathrm{~km} \cdot \mathrm{hr}^{-1}\right)$ & $\begin{array}{l}\text { Ambulatory } \\
\text { (optional) }\end{array}$ & 5 & 12.62 & 1.17 & 5151 & 1113 & 4844 & 1232 & 3756 & 631 & 8825 & 1592 & 5525 & 787 \\
\hline
\end{tabular}

* listwise sample size

$\dagger$ indicates that participants were randomly allocated 2 out of the 4 activities in this grouping

$\$$ indicates that participants had the option to run (select 1 out of the 3 activities in this grouping) if they so chose

Note: $1 \mathrm{MET}=3.5 \mathrm{ml} \cdot \mathrm{kg} \cdot \mathrm{min}^{-1}$ 
Table 4. Sensitivity, specificity, area under the ROC curve, and GENEA SVMgs ( $\mathrm{g} \cdot$ minutes) cut points that maximized sensitivity and specificity at three wear positions

\begin{tabular}{|c|c|c|c|c|}
\hline Intensity* & Sensitivity & Specificity & $\begin{array}{l}\text { Area under ROC } \\
\text { curve }(95 \% \mathrm{CI})\end{array}$ & $\begin{array}{l}\text { GENEA Cut points } \\
\text { SVMgs }(\mathrm{g} \cdot \mathrm{minutes})\end{array}$ \\
\hline \multicolumn{5}{|l|}{ Left Wrist } \\
\hline Sedentary & 97 & 95 & $0.98(0.98-0.99)$ & $<217$ \\
\hline Light & NA & NA & NA & $217-644$ \\
\hline Moderate & 95 & 72 & $0.91(0.88-0.93)$ & $645-1810$ \\
\hline Vigorous & 78 & 98 & $0.91(0.86-0.95)$ & $>1810$ \\
\hline \multicolumn{5}{|l|}{ Right Wrist } \\
\hline Sedentary & 99 & 96 & $0.98(0.97-0.99)$ & $<386$ \\
\hline Light & NA & NA & NA & $386-439$ \\
\hline Moderate & 100 & 56 & $0.84(0-81-0.87)$ & $440-2098$ \\
\hline Vigorous & 78 & 97 & $0.89(0.84-0.94)$ & $>2098$ \\
\hline \multicolumn{5}{|l|}{ Waist } \\
\hline Sedentary & 99 & 96 & $0.97(0.96-0.98)$ & $<77$ \\
\hline Light & NA & NA & NA & $77-219$ \\
\hline Moderate & 96 & 80 & $0.93(0.91-0.95)$ & $220-2056$ \\
\hline Vigorous & 73 & 99 & $0.92(0.88-0.96)$ & $>2056$ \\
\hline
\end{tabular}




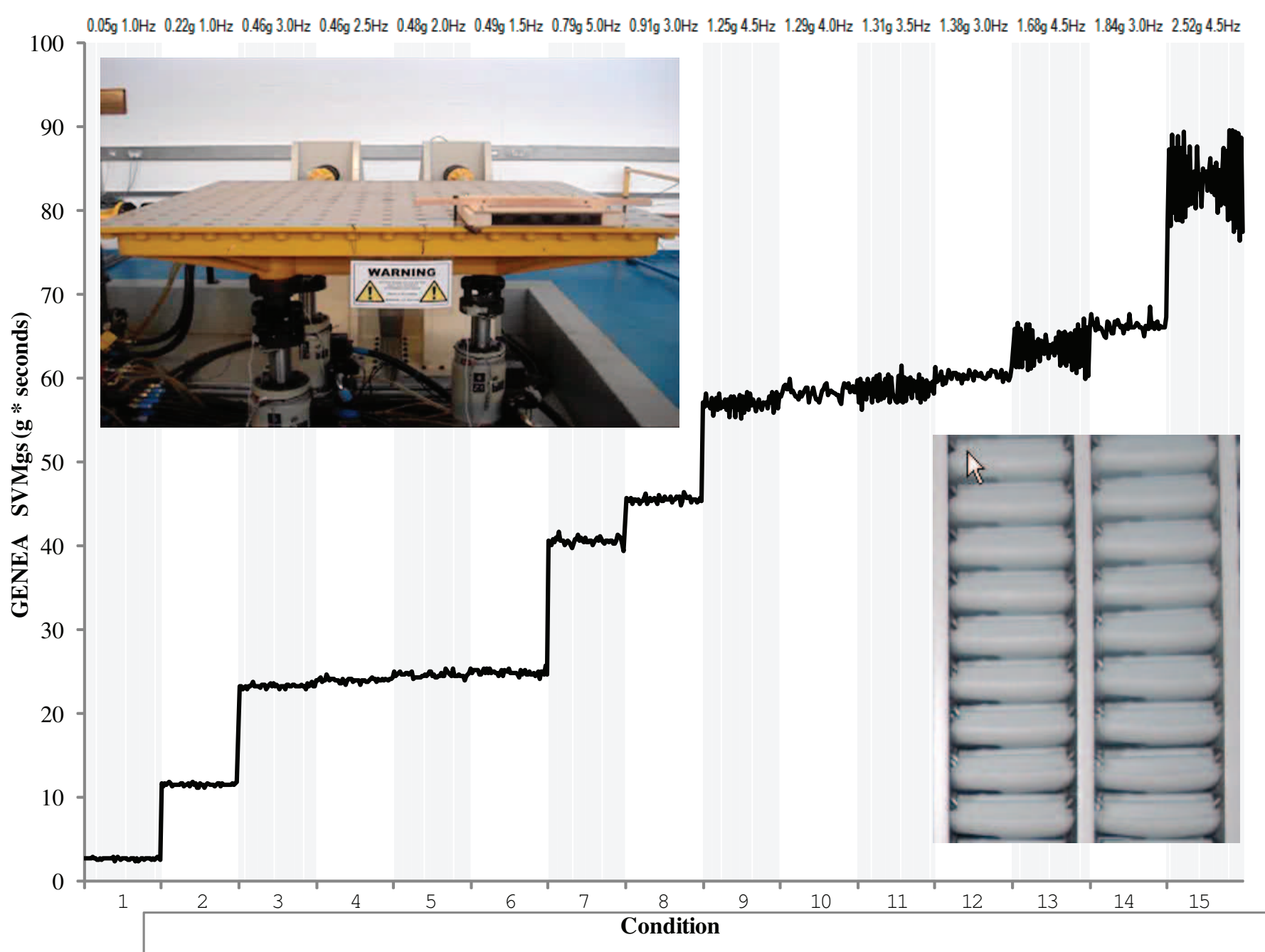


